\title{
Differential Effects of Fingolimod and Natalizumab on B Cell Repertoires in Multiple Sclerosis Patients
}

\author{
M. C. Kowarik ${ }^{1,2}$ - D. Astling ${ }^{3} \cdot$ G. Lepennetier ${ }^{2} \cdot$ A. Ritchie $^{4} \cdot$ B. Hemmer ${ }^{2,5} \cdot$ G. P. Owens ${ }^{4} \cdot$ Jeffrey L. Bennett ${ }^{4,6}$ (I)
}

Accepted: 14 November 2020 / Published online: 30 November 2020

(C) The American Society for Experimental NeuroTherapeutics, Inc. 2020

\begin{abstract}
Natalizumab and fingolimod are effective multiple sclerosis (MS) therapies that disrupt lymphocyte migration but have differential effects on B cell maturation and trafficking. We investigated their effects on peripheral blood (PB) and cerebrospinal fluid (CSF) B cell repertoires using next-generation deep sequencing. Paired CSF and PB B cell subsets (naïve, CD27 $7^{+}$memory, and $\mathrm{CD}_{27}{ }^{-} \mathrm{IgD}^{-}$double-negative B cells and plasmablasts) were collected by applying flow cytometry at baseline and after 6 months of treatment and their respective heavy-chain variable region repertoires assessed by Illumina MiSeq. Treatment with fingolimod contracted, whereas natalizumab expanded circulating PB B cells. CSF B cell numbers remained stable following fingolimod treatment but decreased with natalizumab therapy. Clonal overlap between CSF and PB B cells was reduced with natalizumab treatment but remained stable with fingolimod therapy. Lineage analyses of pre- and posttreatment CSF B cell repertoires revealed large, clonally expanded B cell clusters in natalizumab-treated MS patients but no intrathecal clonal expansion following fingolimod therapy. Our findings suggest that natalizumab diminishes the exchange of peripheral and intrathecal B cells without impacting intrathecal clonal expansion. In contrast, fingolimod treatment fails to alter blood-brain barrier B cell exchange but diminishes intrathecal clonal expansion. Sphingosine-1 phosphate receptor inhibition may alter intrathecal B cell biology in MS.
\end{abstract}

Key Words Natalizumab $\cdot$ fingolimod $\cdot$ cerebrospinal fluid $\cdot B$ cell migration $\cdot B$ cell maturation $\cdot$ mass sequencing.

\section{Introduction}

B cells play an important role in the pathophysiology of multiple sclerosis (MS). B cells are detected in MS lesions and

Jeffrey L. Bennett

jeffrey.bennett@cuanschutz.edu

1 Department of Neurology \& Stroke and Hertie-Institute for Clinical Brain Research, Eberhard-Karls University of Tübingen, Hoppe-Seyler-Str. 3, 72076 Tübingen, Germany

2 Department of Neurology, Technische Universität München, Ismaninger Str. 22, 81541 Munich, Germany

3 Department of Biochemistry and Molecular Genetics, University of Colorado, 13001 East 17th Place, Aurora, Colorado 80045, USA

4 Department of Neurology, University of Colorado Anschutz Medical Campus, 12700 East 19th Avenue, Aurora, Colorado 80045, USA

5 Munich Cluster for Systems Neurology (SyNergy), Feodor-Lynen-Str. 17, 81377 Munich, Germany

6 Department of Ophthalmology, Programs in Neuroscience and Immunology, University of Colorado Anschutz Medical Campus, 12700 East 19th Avenue, Aurora, Colorado 80045, USA cerebrospinal fluid (CSF), and the persistence of intrathecal oligoclonal immunoglobulin $\mathrm{G}$ (IgG) bands is a fundamental hallmark of disease and associated with worsening disability $[1,2]$. Phase 3 clinical trials have demonstrated that B celldepleting therapies are effective in reducing clinical and magnetic resonance imaging (MRI) activity in relapsing and progressive forms of disease [3-5].

Oral fingolimod and natalizumab are MS therapeutics that interfere with lymphocyte migration and exert distinct effects on B cell trafficking [6, 7]. Fingolimod is a structural analogue to sphingosine and acts as a superagonist for the sphingosine-1phosphate receptor 1 (S1P1) on lymphocytes [8], thus inhibiting the egress of lymphocytes from secondary lymphoid tissues. Fingolimod treatment produces a significant peripheral lymphopenia with decreased B cells [9]; the CSF B cell fraction, however, remains elevated [10]. Natalizumab, a monoclonal antibody against $\alpha 4$-integrin, reduces the ability of activated $T$ cells to migrate through the blood-brain barrier, producing significantly reduced B cell numbers in the CSF [11]. Following natalizumab treatment, peripheral blood B cells are increased due to the release of memory B cells that are normally attached to the marginal sinus of the spleen via alpha 4 integrins [12]. 
The purpose of this study was to compare and contrast CNS B cell trafficking and distribution in natalizumab- and fingolimod-treated MS patients using massively parallel DNA sequencing of immunoglobulin heavy-chain $(\mathrm{VH})$ repertoires. In total, 8 patients receiving either natalizumab or fingolimod were studied at baseline (T0) and following 6 months (T6) of treatment. At each time point, CSF and peripheral blood B cells were sorted into naïve, memory, double-negative, and plasmablast populations and VH repertoires assessed by next-generation mass sequencing. Comparison of Ig repertoires between the peripheral blood and CSF compartments at baseline and over time indicated that natalizumab treatment diminished but did not completely block the migration of B cells into the CSF. In contrast, fingolimod significantly reduced peripheral blood B cell numbers and CSF B cell clonal expansion without diminishing B cell migratory potential.

\section{Methods}

\section{Standard Protocol Approvals, Registrations, and Patients}

Patients with relapsing remitting multiple sclerosis were recruited into the study at the University of Colorado, School of Medicine, from 2013 to 2014 following Institutional Review Board-approved informed consent. Study inclusion criteria were the following: 1) diagnosis of clinically definite MS according to International (McDonald 2011) or Poser criteria; 2) age between 18 and 65 years of age; 3 ) the ability to give informed consent; and 4) normal vital signs. Exclusion criteria included 1) CNS disease in addition to MS; 2) primary progressive or secondary progressive forms of MS; 3 ) bacterial or viral infection within the last 30 days; 4) prior immunomodulatory or immunosuppressant therapy azathioprine, methotrexate, cyclophosphamide, mitoxantrone, rituximab, daclizumab, mycophenolate mofetil, laquinimod, or total body irradiation; and 5) treatment within the last 30 days with corticosteroids, beta-interferon, glatiramer acetate, plasma exchange, or intravenous immunoglobulin. The planned analysis was for 8 patients: 4 natalizumab and 4 oral fingolimod. Nine patients were recruited; a single patient withdrew from participation after initial lumbar puncture. Treatment with oral fingolimod or natalizumab was initiated at the discretion of the patient's treating physician. The study duration was 6 months with peripheral blood and CSF obtained at study entry and exit.

\section{Specimen Handling and Routine CSF Testing}

CSF and blood (CPT tubes; BD Vacutainer) were collected according to standard procedures at the same study visit from patients at baseline and after 6 months of treatment with fingolimod or natalizumab using standard dosing. We collected two CPT tubes (16 ml whole blood) and one CSF tube containing $8 \mathrm{ml} \mathrm{CSF}( \pm 1 \mathrm{ml})$ at each time point. Routine CSF and serum analyses (Table 1) showed no significant differences between treatment groups.

\section{Cell Staining and Sorting of CSF and Blood B Cell Populations}

CSF cells and blood mononuclear cells (MNCs) were collected by centrifugation, washed with phosphate-buffered saline (PBS), and suspended in PBS containing 5\% fetal calf serum. Cells were then incubated ( $40 \mathrm{~min}$ ) on ice with antibodies to the following cell markers: CD27 PE (Becton Dickinson, FranklinLakes, NJ), CD38 APCCy5.5 (Invitrogen, Carlsbad, CA), CD19 PacBlue (AbDserotec, Oxford, United Kingdom), IgD APC (Becton Dickinson), CD14 FITC (AbDserotec), CD56 FITC (Becton Dickinson), CD20 FITC (Biolegend), CD16 FITC (eBioscience, San Diego, CA), and CD3 FITC (Becton Dickinson). After staining, cells were washed and suspended in PBS for further flow cytometric analysis and cell sorting on a MoFlo cytometer (Cytomations, Fort Collins, CO). $\mathrm{CD} 19^{+} \mathrm{CD} 20^{+}$and $\mathrm{CD} 19^{+} \mathrm{CD} 20^{\text {low }} \mathrm{B}$ cell subsets (Suppl. Fig. 1) were first selected from other cell populations as previously described [13]. Naïve B cells $\left(\mathrm{CD} 19^{+} \mathrm{CD} 20^{+} \mathrm{IgD}{ }^{+} \mathrm{CD} 27^{-}\right)$ and memory $\mathrm{B}$ cells $\left(\mathrm{CD} 19^{+} \mathrm{CD} 20^{+} \mathrm{CD} 27^{+}\right)$were further separated from the $\mathrm{CD} 19^{+} \mathrm{CD} 20^{+}$population, whereas doublenegative $\mathrm{B}$ cells $\left(\mathrm{CD} 19^{+} \mathrm{CD} 20^{\text {low }} \mathrm{CD} 27^{-} \mathrm{IgD}^{-} \mathrm{CD} 38^{+}\right)$and plasmablasts $\left(\mathrm{CD} 19^{+} \mathrm{CD} 20^{\text {low }} \mathrm{CD} 27^{+} \mathrm{CD} 38^{\text {high }} \mathrm{IgD}^{-}\right)$were obtained from the $\mathrm{CD} 19^{+} \mathrm{CD} 20^{\text {low }}$ population. The gating strategy (Suppl. Fig. 1) and numbers of PB and CSF B cells collected from each population per subject (Suppl. Table 1A, B) are provided in supplementary materials.

\section{CSF and Blood VH Transcriptome Library Preparation and Deep Sequencing}

Total RNA was extracted from peripheral blood and CSF B cell populations using the Qiagen RNeasy Plus Micro Kit (Qiagen, Germantown, MD; 74004) per manufacturer's instructions. cDNA was synthesized using the Clontech SMARTer Ultra Low RNA Kit (Takara Bio USA, Mountain View, CA; 634936) for Illumina sequencing according to the manufacturer's instructions, containing an amplification step during second-strand cDNA synthesis. During the doublestranded cDNA synthesis step, conserved constant region primers for IgA, IgG, and IgM were added to enrich immunoglobulin transcripts during amplification [13]. Following generation of ds cDNA libraries, a pool of modified, $\mathrm{VH}$ family-specific (VH1-VH5) and isotype-specific (IgD, IgM, $\mathrm{IgG}$, and $\operatorname{IgA}$ ) nested $\mathrm{C}$ region primers were used to amplify $\mathrm{VH}$ region sequences using polymerase chain reaction (PCR high fidelity, Roche, Indianapolis, IN). Separate PCR 
Table 1 Routine MS patient CSF and serum analysis at baseline and 6-months after treatment with either fingolimod or natalizumab

\begin{tabular}{|c|c|c|c|c|c|c|c|c|}
\hline & \multicolumn{2}{|c|}{ Subject 2} & \multicolumn{2}{|c|}{ Subject 3} & \multicolumn{2}{|c|}{ Subject 4} & \multicolumn{2}{|c|}{ Subject 8} \\
\hline Age (yr), gender & \multicolumn{2}{|c|}{$52, \mathrm{~F}$} & \multicolumn{2}{|c|}{$43, \mathrm{~F}$} & \multicolumn{2}{|c|}{$57, \mathrm{~F}$} & \multicolumn{2}{|c|}{$53, \mathrm{~F}$} \\
\hline Disease duration (mo) & \multicolumn{2}{|l|}{53} & \multicolumn{2}{|l|}{95} & \multicolumn{2}{|l|}{98} & \multicolumn{2}{|l|}{168} \\
\hline Time since relapse (mo) & \multicolumn{2}{|l|}{14} & \multicolumn{2}{|l|}{24} & \multicolumn{2}{|l|}{54} & \multicolumn{2}{|l|}{6} \\
\hline Prior MS therapies & \multicolumn{2}{|l|}{ None } & \multicolumn{2}{|c|}{ INF, GLAT, NAT } & \multicolumn{2}{|l|}{ GLAT } & \multicolumn{2}{|c|}{ INF, GLAT, NAT, DMF } \\
\hline Time since last $\mathrm{Tx}(\mathrm{mo}, \mathrm{Tx})$ & \multicolumn{2}{|l|}{ N/A } & \multicolumn{2}{|c|}{14, NAT } & \multicolumn{2}{|c|}{ 1, GLAT } & \multicolumn{2}{|c|}{$11, \mathrm{DMF}$} \\
\hline \multirow[t]{2}{*}{ EDSS } & 1 & & 1.5 & & 1.5 & & 3 & \\
\hline & Pre-Rx & Fingolimod & Pre-Rx & Fingolimod & Pre-Rx & Fingolimod & Pre-Rx & Fingolimod \\
\hline CSF glucose & 59 & 57 & 56 & 54 & ND & 64 & 56 & 63 \\
\hline CSF protein & 36 & 29 & 54 & 50 & 24 & 24 & 21 & 27 \\
\hline WBC & 0 & 4 & 3 & 0 & 0 & 0 & 0 & 0 \\
\hline Ser IgG (mg/dl) & 749 & 667 & 568 & 753 & 952 & 946 & 637 & 652 \\
\hline Ser Alb (mg/dl) & 3970 & 4480 & 4340 & 4100 & 4220 & 4340 & 4770 & 4450 \\
\hline CSF Alb (mg/dl) & 22 & 22 & 34 & 31 & 14 & 17 & 15 & 17 \\
\hline Albumin index & 5.5 & 4.9 & 7.8 & 7.6 & 3.3 & 3.9 & 3.1 & 3.8 \\
\hline IgG CSF (mg/dl) & 3 & 2.6 & 5.3 & 5.1 & 1.7 & 1.7 & 1.2 & 1.3 \\
\hline IgG Index & 0.72 & 0.79 & 1.19 & 0.9 & 0.54 & 0.46 & 0.6 & 0.52 \\
\hline $\mathrm{IgG} / \mathrm{Alb}$ ratio & 0.14 & 0.12 & 0.16 & 0.16 & 0.12 & 0.1 & 0.08 & 0.08 \\
\hline OCB \# of bands & 13 & 10 & 15 & 12 & 0 & 0 & 1 & 1 \\
\hline IgG synthesis & 3 & 3.4 & 14.4 & 10 & 0 & 0 & 0 & 0 \\
\hline & Subject & & Subject & & Subject & & Subject & \\
\hline Age (yr), gender & $20, \mathrm{~F}$ & & $35, \mathrm{M}$ & & $22, \mathrm{~F}$ & & $39, \mathrm{~F}$ & \\
\hline Disease duration (mo) & 2 & & 4 & & 115 & & 42 & \\
\hline Time since relapse (mo) & 2 & & 4 & & 12 & & 2 & \\
\hline Prior MS therapies & None & & None & & GLAT & & GLAT, & DMF \\
\hline Time since last $\mathrm{Tx}(\mathrm{mo}, \mathrm{Tx})$ & N/A & & N/A & & 1, GLA $^{\prime}$ & & $5, \mathrm{DMF}$ & \\
\hline EDSS & 2.5 & & 2.5 & & 1.5 & & 2.5 & \\
\hline & Pre-Rx & Natalizumab & Pre-Rx & Natalizumab & Pre-Rx & Natalizumab & Pre-Rx & Natalizumab \\
\hline CSF glucose & 54 & 55 & 56 & 55 & 54 & 53 & 52 & 62 \\
\hline CSF protein & 32 & 21 & 68 & 50 & 27 & 26 & 29 & 38 \\
\hline WBC & 3 & 0 & 14 & 2 & 11 & 2 & 2 & 2 \\
\hline Ser IgG (mg/dl) & 935 & 559 & 775 & 946 & 855 & 719 & 1120 & 1120 \\
\hline Ser Alb (mg/dl) & 4470 & 3130 & 4870 & 4360 & 4580 & 4730 & 3150 & 4010 \\
\hline CSF Alb (mg/dl) & 18 & 15 & 56 & 35 & 19 & 18 & 17 & 17 \\
\hline Albumin index & 4 & 4.8 & 11.5 & 8 & 4.1 & 3.8 & 5.4 & 4.2 \\
\hline IgG CSF (mg/dl) & 3.1 & 1.7 & 9 & 6.9 & 3.2 & 2.4 & 8.7 & 7.6 \\
\hline IgG Index & 0.82 & 0.63 & 1.01 & 1.14 & 0.9 & 0.88 & 1.44 & 1.6 \\
\hline $\mathrm{IgG} / \mathrm{Alb}$ ratio & 0.17 & 0.11 & 0.16 & 0.2 & 0.17 & 0.13 & 0.51 & 0.45 \\
\hline OCB \# of bands & 6 & 7 & 17 & 13 & 19 & 10 & 17 & 15 \\
\hline IgG synthesis & 3.4 & 0.6 & 22.5 & 18.2 & 5 & 2.9 & 25.8 & 26.2 \\
\hline
\end{tabular}

There were no significant differences between treatment groups in baseline serum and CSF values (Mann-Whitney $U$ test)

$\mathrm{WBC}=$ white blood count Ser = serum $; \mathrm{CSF}=$ cerebrospinal fluid $; \mathrm{OCB}=$ oligoclonal bands; Pre $-\mathrm{Rx}=$ baseline; $\mathrm{MS}=$ multiple sclerosis; $\mathrm{yr}=\mathrm{years} ; \mathrm{mo}$ $=$ months; $\mathrm{Tx}=$ treatment; GLAT $=$ glatiramer; $\mathrm{INF}=$ interferon; $\mathrm{NAT}=$ natalizumab; $\mathrm{DMF}=$ dimethyl fumarate

reactions were performed for each VH family to avoid crosspriming or primer competition. Each nested Ig constant region primer contained a sequence tag ("barcode") to identify the specific patient B cell population of origin and Ig isotype, a random sequence insert (unique molecular identifier; UMI) to identify PCR overreplication [14], and a unique shared primer sequence for subsequent Illumina MiSeq deep sequencing. Forward primers contained a primer sequence for sense strand sequencing. Amplified cDNA from peripheral blood and CSF $B$ cell subsets of each subject and from each time point (before 
Fig. 1 Distribution of peripheral blood (PB) and cerebrospinal fluid (CSF) B cell subtypes, shown as the delta change of sorted cells after 6 months of treatment with (a) fingolimod $(n=4)$ or (b) natalizumab $(n=4)$ compared to baseline. The sorting paradigm for naïve, memory, doublenegative (DN), and plasmablast populations are defined in the "Methods" section. Values are presented as the absolute difference in recovered cells from baseline for each B cell subset. The number above each treatment indicates the fold change in that $\mathrm{B}$ cell subset relative to the untreated cohort. Statistical significance could not be established due to limited patient numbers

\section{a Fingolimod}

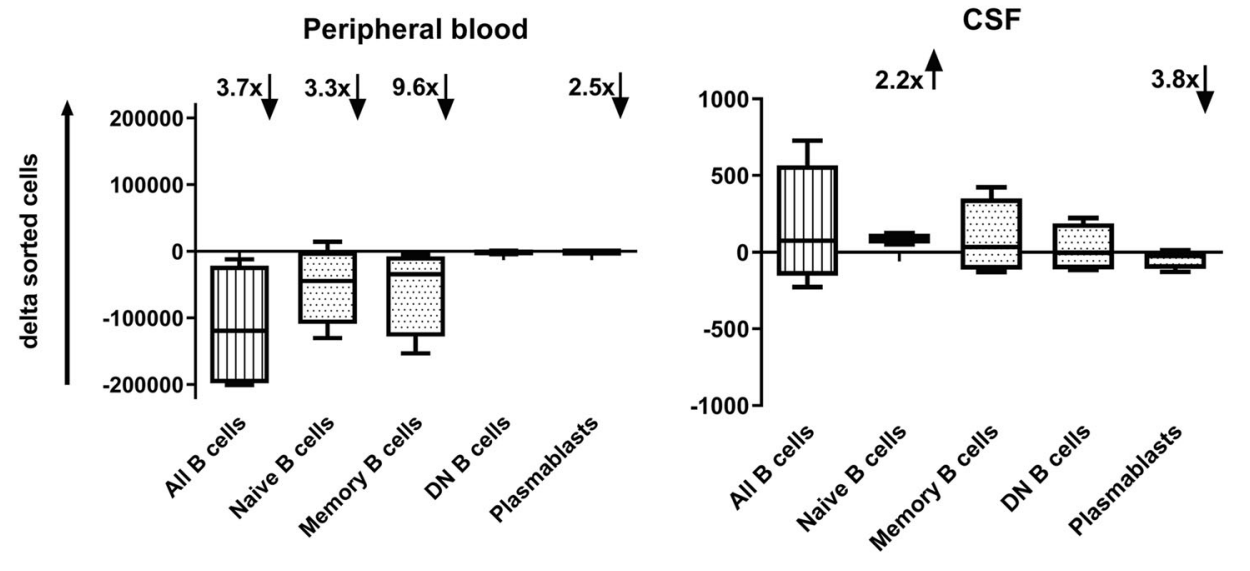

\section{b Natalizumab}

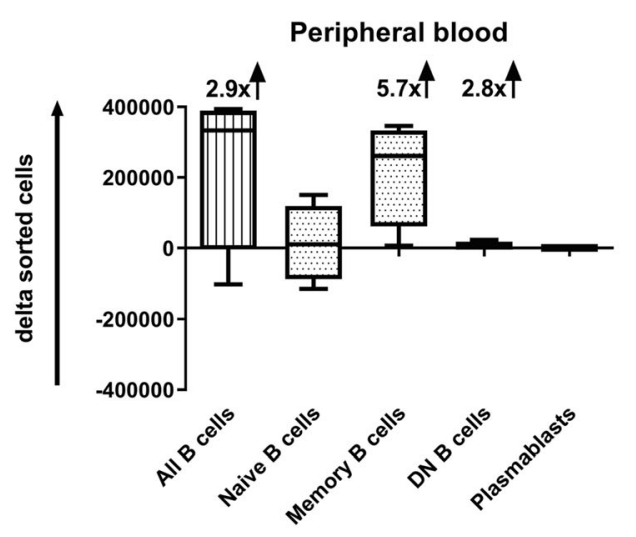

and after treatment) were separately pooled and sequenced in a single run on an Illumina MiSeq Personal Sequence yielding 16 individual VDJ libraries from the $8 \mathrm{MS}$ patients. Our approach allowed sequencing of the entire heavy-chain variable region with a maximum read of $600 \mathrm{bp}$.

\section{Data Analysis}

According to their barcodes, Illumina sequencing reads in FASTQ format were presorted into patient-specific B cell populations. Illumina MiSeq high-throughput sequencing reads were quality-controlled, assembled, and filtered using pRESTO [15]. The workflow took advantage of our unique molecular identifiers (UMIs) to eliminate redundant reads from the same PCR priming event.

The complementarity determining region-3 (CDR3) of the $\mathrm{VH}$ gene results from the recombination of the variable, diversity, and joining $[\mathrm{V}(\mathrm{D}) \mathrm{J}]$ germline segments. Functional heavychain $[\mathrm{V}(\mathrm{D}) \mathrm{J}]$ germline segments were determined using IMGT/HighV-QUEST [16] and VH sequences assigned into clones based on 1) identical nucleic acid CDR3 sequence length,
2) identical V and J gene segments, and 3) $80 \%$ nucleotide CDR3 sequence identity. From identified clonal groups within the same patient populations, lineage analysis (rooted B cell lineage trees) was performed using IgTree [17] (kindly provided by Prof. Ramit Mehr, Bar-Ilan University, Tel Aviv) to sort clones based on their mutational distance from germline. Alignments were initiated at nucleotide 75 . The germline used as the root of each clonal tree was determined by automatically searching for the best match of the $\mathrm{V}$ and $\mathrm{J}$ alleles from the IgBlast genes (Blastn v2.2.31), concatenating both alleles into a sequence (G) that was then aligned to each member of the clonal group. Because the D segment is often difficult to determine, we filled in the missing gaps of the CDR3 region in the sequence (G) by Ns in order to avoid hypervariable region bias in IgTree.

Each clonal group tree was then converted to a graphml file using in-house conversion tools and improved via Cytoscape functionalities. The program GNU parallel was used at multiple steps in the assembly of full-length VDJ sequences. Using the output of IgTree, it was also possible to evaluate the relatedness of Ig transcripts over time (T0 vs. T6) and across compartments (peripheral blood $v s$. CSF). 


\section{Protocol for Tree Sorting and Detailed Lineage Analyses}

The IgTree program produces, in addition to numeric images, ".vsdot" files. Those files contain descriptive information for each individual tree. Using in-house python scripts (available under https://github.com/GildasLepennetier/TreeSelect), we extracted the tree structure and composition. All trees were then automatically sorted step by step as described in Suppl. Table 2 and then manually refined. After the refinement, nodes in the lineage tree were labeled based on the compartment (peripheral blood or CSF) and B cell subtype of the most frequent sequence in that clonal group.

\section{Statistics}

Wilcoxon signed-rank test (one-sided) for paired samples was used to test for significance between different time points within treatment groups. The Mann-Whitney $U$ test was used to test for significant differences between treatment groups. For comparison of different B cell subsets the nonparametric Kruskal-Wallis test with multiple comparison correction (Dunn's procedure) was applied.

\section{Results}

\section{Clinical and Laboratory Data}

Nine patients from the Rocky Mountain Multiple Sclerosis Center at Anschutz Medical Campus were recruited for participation based on inclusion/exclusion criteria and signed informed consent. One patient withdrew after the initial lumbar puncture. MS treatment was predetermined by the patient's treating physician. Two natalizumab patients and one fingolimod patient were treatment naïve; all initiated therapy within roughly 1 year of their initial presentation. The remaining five patients had received prior MS treatments including beta-interferon, glatiramer acetate, natalizumab, and dimethyl fumarate. For the two patients who previously received natalizumab, their last infusions were 14 months and 60 months, prior to study entry. For the two patients who previously received dimethyl fumarate, the medication was discontinued 5 months and 11 months prior to study entry after 5 and 28 days of therapy, respectively. Five patients ( 3 fingolimod and 2 natalizumab) switched therapy due to disease activity. Two changed treatments after clinical relapses (2 and 6 months, respectively), whereas the others switched due to new disease activity on MRI. Both relapses occurred while off of therapy. No patients experienced a clinical relapse during the 6-month study period. All standard of care brain MRIs performed 12 months after treatment did not show any new lesion activity (further clinical data Table 1). Although 2 patients from the natalizumab treatment group showed a mild CSF pleocytosis at the onset of treatment, there were no significant differences in any baseline CSF parameters between treatment groups. There were no significant differences in the changes from baseline in any CSF parameter between treatments (Table 1).

Due to their differing modes of action, distinct treatmentspecific changes were noted in peripheral blood B cell subsets (Fig. 1). Fingolimod treatment caused a decrease in the

Table 2 Clonal analyses of Ig repertoires within the peripheral blood and cerebrospinal fluid of treatment cohorts

\begin{tabular}{|c|c|c|c|c|c|}
\hline & \multicolumn{2}{|l|}{ Fingolimod } & \multicolumn{2}{|l|}{ Natalizumab } & \multirow{2}{*}{$\begin{array}{l}\text { Fingolimod } \\
\text { vs. natalizumab } \\
\text { T6 }\end{array}$} \\
\hline & T0 & T6 & T0 & T6 & \\
\hline \multicolumn{6}{|l|}{ CSF sequences } \\
\hline$\%$ CSF sequences in clones/SDM & $\begin{array}{l}71 \% / \pm 27 \% \\
p=\mathrm{NS}\end{array}$ & $77 \% / \pm 13 \%$ & $\begin{array}{l}89 \% / \pm 3 \% \\
p=\mathrm{NS}\end{array}$ & $82 \% / \pm 8 \%$ & $p=\mathrm{NS}$ \\
\hline Average \# of clones/SDM & $\begin{array}{l}\text { 13/ } \pm 9 \\
\text { T0 vs. T6: } p=\mathrm{NS}\end{array}$ & $28 / \pm 31$ & $\begin{array}{l}221 / \pm 176 \\
\text { T0 vs. T6: } p=0.06\end{array}$ & $18 / \pm 6$ & $p=\mathrm{NS}$ \\
\hline Average \# of sequences per clone/SDM & $\begin{array}{l}44 / \pm 38 \\
\text { T0 vs. T6: } p=\mathrm{NS}\end{array}$ & $18 / \pm 9$ & $\begin{array}{l}17 / \pm 5 \\
\text { T0 vs. T6: } p=0.06\end{array}$ & $35 / \pm 9$ & $p=\mathrm{NS}$ \\
\hline \multicolumn{6}{|l|}{ PB sequences } \\
\hline$\% \mathrm{~PB}$ sequences in clones/SDM & $\begin{array}{l}46 \% / \pm 9 \% \\
\text { T0 vs. T6: } p=\mathrm{NS}\end{array}$ & $74 \% / \pm 19 \%$ & $\begin{array}{l}54 \% / \pm 7 \% \\
\text { T0 vs. T6: } p=0.06\end{array}$ & $38 \% / \pm 4 \%$ & $p=0.03$ \\
\hline Average \# of clones/SDM & $\begin{array}{l}\text { 1697/ } \pm 683 \\
\text { T0 vs. T6: } p=\mathrm{NS}\end{array}$ & $447 / \pm 136$ & $\begin{array}{l}3727 / \pm 2526 \\
\text { T0 vs. T6: } p=\mathrm{NS}\end{array}$ & $4122 / \pm 1502$ & $p=0.03$ \\
\hline Average \# of sequences per clone/SDM & $\begin{array}{l}4 / \pm 1 \\
\text { T0 vs. T6: } p=\mathrm{NS}\end{array}$ & $10 / \pm 4$ & $\begin{array}{l}4 / \pm 1 \\
\text { T0 vs. T6: } p=\mathrm{NS}\end{array}$ & $4 / \pm 0$ & $p=0.03$ \\
\hline
\end{tabular}

Wilcoxon signed-rank test (paired samples) was used to test between different time points, Mann-Whitney $U$ test was used to test between treatment groups

$\mathrm{SDM}=$ standard deviation of the mean; $\mathrm{CSF}=$ cerebrospinal fluid; $\mathrm{PB}=$ peripheral blood; $\mathrm{T} 0=$ baseline; $\mathrm{T} 6=6$ months of treatment; $N S=$ not significant 
fraction of recovered $\mathrm{CD} 19^{+} \mathrm{B}$ cells predominantly within the naïve (3.3-fold), plasmablast (2.5-fold), and memory B cell subsets (9.6-fold); DN B cells were marginally affected. Conversely, natalizumab treatment increased the number of recovered $\mathrm{B}$ cells with increases observed in the memory (5.7-fold) and DN B cell pools (2.8-fold). Within the CSF, the number of recovered B cells did not change appreciably from baseline following fingolimod therapy (Suppl. Table 2). A modest increase in the fraction of naïve B cells (2.2-fold) and a decrease in plasmablasts (3.8-fold) were observed (Fig. 1). Natalizumab treatment, however, produced a 2 -fold reduction in B cell recovery with memory B cells (3.1-fold) and plasmablasts (6-fold) most affected.

\section{Ig Repertoires Generated from Blood and CSF B Cell Subsets}

B cell clonal expansion is observed within the CSF compartment of MS patients. To assess changes in the clonal relationships within and between CSF and blood B cells following therapy, B cell subsets (naïve, memory, and double-negative B cells and plasmablasts) were sorted and functional heavychain ( $\mathrm{VH}$ ) repertoires generated using Illumina nextgeneration deep sequencing (Suppl. Table 1A) in the $8 \mathrm{MS}$ patients. On average, 78,586 sequences from blood B cell subsets (range 20,329-243,433 sequences) and 14,130 sequences from CSF B cell subsets (range 241-102,028 sequences) were processed through the pRESTO bioinformatics pipeline to obtain representative $\mathrm{VH}$ repertoires for each $\mathrm{B}$ cell population (Suppl. Table 1B).

At baseline, the distributions of $\mathrm{VH}$ isotypes in peripheral blood B cell populations were $50 \% \operatorname{IgD}$ and $50 \% \operatorname{IgM}$ in the naïve B cell population; 50\% $\operatorname{IgM}, 30 \% \operatorname{IgA}$, and $20 \% \operatorname{IgG}$ in the memory population; and 26\% IgM, 53\% IgA, and $21 \%$ $\mathrm{IgG}$ in the $\mathrm{DN}$ population (not shown). Plasmablasts were $30 \%$ IgM, 40\% IgA, and 30\% IgG. In the CSF, at baseline, there was a significantly higher fraction of IgG-expressing B cells in the memory, DN, and plasmablast subsets compared to peripheral blood (not shown). There were no significant changes in the distribution of $\mathrm{Ig}$ isotypes in the peripheral blood and CSF subsets following treatment with either medication (Suppl. Fig. 2a).

Prior to the onset of treatment, the VH germline distribution of peripheral blood B cell subsets approximated germline prevalence; the VH3 family was most frequently utilized, followed by the VH1 and VH4 family gene segments [18] (Suppl. Fig. 2b). The baseline VH germline distribution in the CSF, however, showed a significant increase in VH4 family gene segments consistent with independent findings observed at the single-cell level in MS CSF plasmablasts [19]. Significantly increased VH4 germline segment usage was accompanied by a reduced presence of VH1 and VH5 family gene segments. Treatments had no discernable effects on $\mathrm{VH}$ family distributions in peripheral blood and CSF (Suppl. Fig. 2b). In the CSF, there was a significant increase in Ig class-switched B cells relative to peripheral blood that was accompanied by sharp declines in CSF IgM- and IgDexpressing B cells (Suppl. Fig. 2a). Consistent with these results, we observed a significantly lower diversity index for CSF B cells when compared to those in the periphery (Suppl. Fig. 2c); however, the assessment of the $\mathrm{VH}$ repertoire diversity in the peripheral blood is likely undervalued by a more limited sampling of this compartment.

\section{Changes in PB and CSF VH Repertoires Following Treatment}

We established representative CSF and PB B cell repertoires at baseline (T0) and after 6 months (T6) of treatment in 3 of 4 fingolimod-treated and all 4 natalizumab-treated patients. The recovery of peripheral blood VH sequences represented on average $9.8 \%$ of sorted cells, whereas in CSF, sequence yields were much higher at $75.3 \%$. The increased sequencing efficiency observed in the CSF B cell population likely reflects the increased prevalence of activated, classswitched memory B cells and plasmablasts containing increased levels of immunoglobulin transcripts. Despite multiple quality steps, it is possible that the proportion of $\mathrm{VH}$ sequences recovered in the CSF may result from overamplification of very low numbers of CSF B cell, thus skewing the $\mathrm{VH}$ repertoire distribution.

Within the CSF compartment, approximately $80 \%$ of $\mathrm{VH}$ sequences were within clonal populations (Table 2). Further comparisons were precluded due to considerable variability in the number and average size of clonal populations amongst different CSF B cell subsets at T0. Overall, the percentage of total CSF VH sequences in clonal populations remained stable following treatment. In fingolimod-treated patients, an increase in the average number of clonal populations was accompanied by a decrease in average clone size, whereas in natalizumab-treated patients, a decrease in the average number of clonal populations was accompanied by a trend towards an increase in average clonal size (Table 2, differences did not reach significance).

Following treatment, there were considerable changes in the composition of peripheral blood $\mathrm{VH}$ repertoires (Table 2). At baseline, approximately $50 \%$ of peripheral blood VH sequences were found within clonal groups with an average clone size of 4 sequences. Following fingolimod treatment, the percentage of peripheral blood $\mathrm{VH}$ sequences within clones increased to $74 \%$ with an average clone size of 10 sequences, whereas following natalizumab treatment, the percentage of clonal $\mathrm{VH}$ sequences in the peripheral blood reduced this to $38 \%$ with a stable clone size of 4 sequences. 

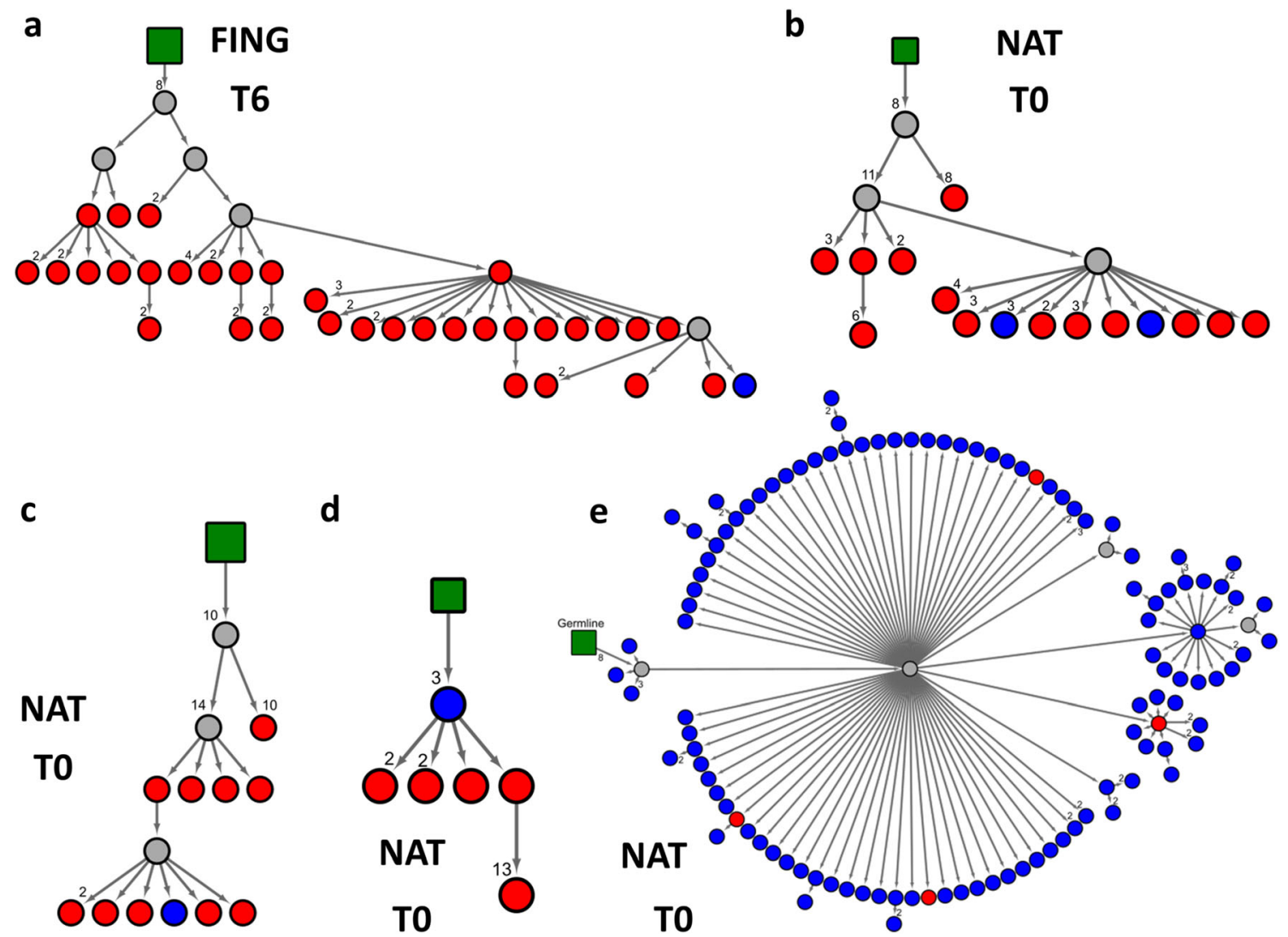

Fig. 2 Lineage trees of representative clonal groups shared between the peripheral blood (PB) and cerebrospinal fluid (CSF) at baseline (T0) and following treatment (T6). Red circles represent PB B cells, blue circles depict CSF B cells, and gray circles indicate putative intermediates originating from the germline sequence (green square). The number adjacent to each arrow denotes the number of mutations between members of the lineage tree. The absence of a number specifies a single mutation. Maturation trees primarily expanded within the peripheral blood (PB)

\section{Clonal Relationships Between Peripheral Blood (PB) and CSF Repertoires at Baseline and 6 Months Following Treatment}

The prevalence of CSF VH sequences identified in paired peripheral blood repertoires at the onset of therapy was $34.8 \%$ in fingolimod-treated and $30.5 \%$ in natalizumabtreated patients (range 6.5-49.8\%) (Table 3). After 6 months, neither treatment significantly altered this overlap. The percentage of blood VH sequences identified in the CSF was considerably lower, consistent with the increased diversity of the peripheral blood repertoire (Table 3). Comparing between therapies, there was an increase in the fraction of overlapping $\mathrm{PB}$ and CSF VH sequences in fingolimod-treated relative to natalizumab-treated patients (Table 3 ).

We next examined the longitudinal effects of treatment on clonal dynamics within the CSF and PB compartments (Table 3). The percentage of baseline CSF sequences identified following 6 months of treatment was limited following either treatment but was lower in fingolimod-treated subjects

are shown in (a-d), whereas maturation trees that are primarily expanded within the cerebrospinal fluid (CSF) are shown in (e). Direct connections between PB and CSF B cells are shown in trees (a, c-e), whereas in tree (b), an unknown intermediate is presumed to be connecting the $\mathrm{PB}$ and CSF compartment. Tree (e) displays PB B cells that most likely underwent further maturation with the CSF compartment. NAT $=$ natalizumab; FING = fingolimod

(0.3\% vs. 6.2\%). A similar significant treatment-specific effect was noted in the persistence of PB clones. Again, fingolimodtreated subjects displayed a significant longitudinal reduction in overlapping sequences relative to natalizumab-treated subjects $(0.7 \%$ vs. $5.5 \%)$ (Table 3$)$.

\section{Lineage Analysis of Overlapping VH Sequences}

We aligned clonally related blood and CSF VH sequences to their most homologous germline sequence, developed hierarchical maturation diagrams (clonal groups) using IgTree, and found 659 of 1019 clonal groups suitable for a qualitative analysis of clonal relationships amongst B cell subsets and compartments. The succession of B cell subtypes and their patterns of somatic hypermutations were then used to predict the most likely direction of maturation (in or out of the CSF). Overall, 388 clonal comparisons were established between CSF and PB at each time point (Fig. 2) and 271 clonal groups within the CSF between baseline (T0) and 6 months of treatment (T6) (Fig. 3). We observed directional B cell trafficking 
Table 3 Overlap of VH repertoires between and within the peripheral blood and cerebrospinal fluid in treatment cohorts

\begin{tabular}{|c|c|c|c|c|c|}
\hline & \multicolumn{2}{|l|}{ Fingolimod } & \multicolumn{2}{|l|}{ Natalizumab } & \multirow{2}{*}{$\begin{array}{l}\text { Fingolimod } \\
\text { vs. natalizumab } \\
\text { T6 }\end{array}$} \\
\hline & T0 & T6 & T0 & T6 & \\
\hline \multicolumn{6}{|l|}{ Between PB and CSF } \\
\hline$\%$ CSF sequences in clones with $\mathrm{PB} / \mathrm{SDM}$ & $\begin{array}{l}34.8 \% / \pm 9.5 \% \\
\text { T0 vs. T6: } p=\mathrm{NS}\end{array}$ & $29.2 \% / \pm 24.6 \%$ & $\begin{array}{l}30.5 \% / \pm 19.1 \% \\
\text { T0 vs. T6: } p=\mathrm{NS}\end{array}$ & $22.5 \% / \pm 13.0 \%$ & $p=\mathrm{NS}$ \\
\hline$\% \mathrm{~PB}$ sequences in clones with $\mathrm{CSF} / \mathrm{SDM}$ & $\begin{array}{l}2.2 \% / \pm 1.5 \% \\
\text { T0 vs. T6: } p=\mathrm{NS}\end{array}$ & $7.1 \% / \pm 3.1 \%$ & $\begin{array}{l}5.1 \% / \pm 2.9 \% \\
\text { T0 vs. T6: } p=\mathrm{NS}\end{array}$ & $1.5 \% / \pm 0.7 \%$ & $p=0.03$ \\
\hline \multicolumn{6}{|l|}{ Within CSF or PB } \\
\hline$\%$ CSF sequences in clones over time/SDM & $0.3 \% / \pm 0.4 \%$ & & $6.2 \% / \pm 4.8 \%$ & & $p=0.04$ \\
\hline$\%$ PB sequences in clones over time/SDM & $0.7 \% / \pm 0.8 \%$ & & $5.5 \% / \pm 2.6 \%$ & & $p=0.03$ \\
\hline
\end{tabular}

Wilcoxon signed-rank test (paired samples) was used to test between different time points, Mann-Whitney $U$ test was used to test between treatment groups

$\mathrm{SDM}=$ standard deviation of the mean; $\mathrm{CSF}=$ cerebrospinal fluid; $\mathrm{PB}=$ peripheral blood; $\mathrm{T} 0=$ baseline; $\mathrm{T} 6=6$ months of treatment; $N S=$ not significant

with a blood B cell followed by a CSF B cell in $90 \%$ of the trees (Fig. 2, clones a-c, e), whereas $10 \%$ of comparisons indicated a likely CSF precursor (Fig. 2, clone d). We further quantified the size of clonal groups by counting the number of contributing sequences within each compartment and B cell subset to determine the likely site of clonal expansion at $\mathrm{T} 0$ and T6 (Fig. 4, Suppl. Table 3).

We first separately analyzed bicompartmental clonal groups (CG) connecting PB and CSF sequences at baseline or after 6 months of treatment (Fig. 4a-c, Suppl. Table 3). Although fingolimod treatment did not affect the average number of clones shared between CSF and peripheral blood ( $\mathrm{T} 0=14$ and $\mathrm{T} 6=15)$, there was a clear shift towards PB B cells as the major contributor (T0: $60 \%$ of sequences; T6: > $95 \%$ of sequences), which was accompanied by a parallel loss in the contribution of expanded CSF B cells (T0: $n=106$ to T6: $n=18,-85 \%$ ), particularly CSF plasmablasts, to common lineages (Fig. 4b). Together, the data indicate that PB B cell trafficking into the CNS was not outwardly altered by fingolimod therapy, but rather clonal expansion of CSF B cells diminished. Indeed, there were significantly fewer number of average CSF sequences at T6 following fingolimod treatment $(n=18)$ when compared to natalizumab $(n=133$, $p=0.03$ ); the difference at $\mathrm{T} 0$ between groups was not significant (Fig. 4a). Following natalizumab treatment, there was a modest decline in the average number of clones shared between CSF and circulating B cells (T0: $n=46$; T6: $n=35,-$ $24 \%$ ), but with no apparent shift in the compartmental contributions of CSF (T0: $n=183$ to T6: $n=133,-27 \%$ ) or PB sequences (T0: $n=535$ to T6: $n=401,-25 \%$ ). The major site of clonal expansion (PB, CSF or equally in both compartments) and changes following treatment are summarized in Fig. 4c. Following fingolimod treatment, there was an increase in PB expanded clones (from $74 \%$ at $\mathrm{T} 0$ to $91 \%$ at
T6), a decreased contribution of CSF-expanded clones (14\% at $\mathrm{T} 0$ to $5 \%$ at $\mathrm{T} 6$ ), and an overall reduction in clones equally abundant in both compartments (14\% at T0 to $5 \%$ at T6). In natalizumab-treated patients, the distribution of major clonal $\mathrm{B}$ cell populations was rather static and consisted mostly of PB memory and DN B cells and plasmablasts (Fig. 4b, c). CSF plasmablasts were still the major CSF contributor to clonality $(\sim 15 \%)$.

Further evidence for impaired B cell expansion within the CSF during fingolimod treatment is indicated from the longitudinal analysis of shared CSF and B cell maturation trees (Fig. 4d-f, Suppl. Table 4). Following treatment, none of the CSF-expanded clones at $\mathrm{T} 0$ were identified in the CSF at T6, and only a single clone arising from the CSF DN B cell population was identified in the peripheral blood PB DN and memory B cell subsets. Thus, treatment with fingolimod appeared to halt expansion of existing clonal B cell populations within the CSF; some of the remaining clones overlapping with clonal populations were evident in the peripheral blood at T0 (Fig. 4e, f).

In contrast, on average, 4 (total $=11$ ) clonal groups connected the CSF compartment between T0 and T6 following natalizumab treatment with more than $50 \%$ of shared T0 clonal sequences (T0: 330, T6: 168) still present. Furthermore, an average of 10 clonal groups (total $n=30$ ) were found connecting CSF at T0 and the PB at T6 (Fig. 4d) with only a limited number of average clonal sequences in $\mathrm{PB}$ at $\mathrm{T} 6(n=$ 18). CSF plasmblasts and memory and DN B cells, which were the major contributors to clonality at $\mathrm{T} 0$ in the natalizumab-treated cohort, changed only marginally at T6. An equal distribution of clonal expansion was observed between the different time points. These results point towards continued B cell maturation within the CSF compartment in natalizumab-treated patients over time. 
Fig. 3 Lineage trees of representative clonal groups maintained in patients at baseline (T0) and following 6 months of treatment (T6). Peripheral bloodderived (red) and cerebrospinal fluid-derived (blue) B cell sequences present at baseline (circles) and 6 months (triangles) are connected by arrows tracing variable region sequence mutations from germline (green square). The number adjacent to each arrow denotes the number of mutations between members of the lineage tree. The absence of a number specifies a single mutation. (a, b, d) Large, highly branched lineage trees link the CSF compartment at baseline and 6 months following natalizumab treatment (NAT). CSF B cells at baseline connected with a high number of CSF B cells after 6 months therapy. Similar clusters were not seen following fingolimod treatment. (c) Lineage tree depicting CSF B cells connected to peripheral blood (PB) B cells after 6 months of NAT. (e) Lineage tree of PB B cells connected to a single CSF B cell after 6 months of NAT. (f) Lineage tree depicting PB B cells at baseline connected to PB B cells after 6 months of fingolimod treatment (FING)
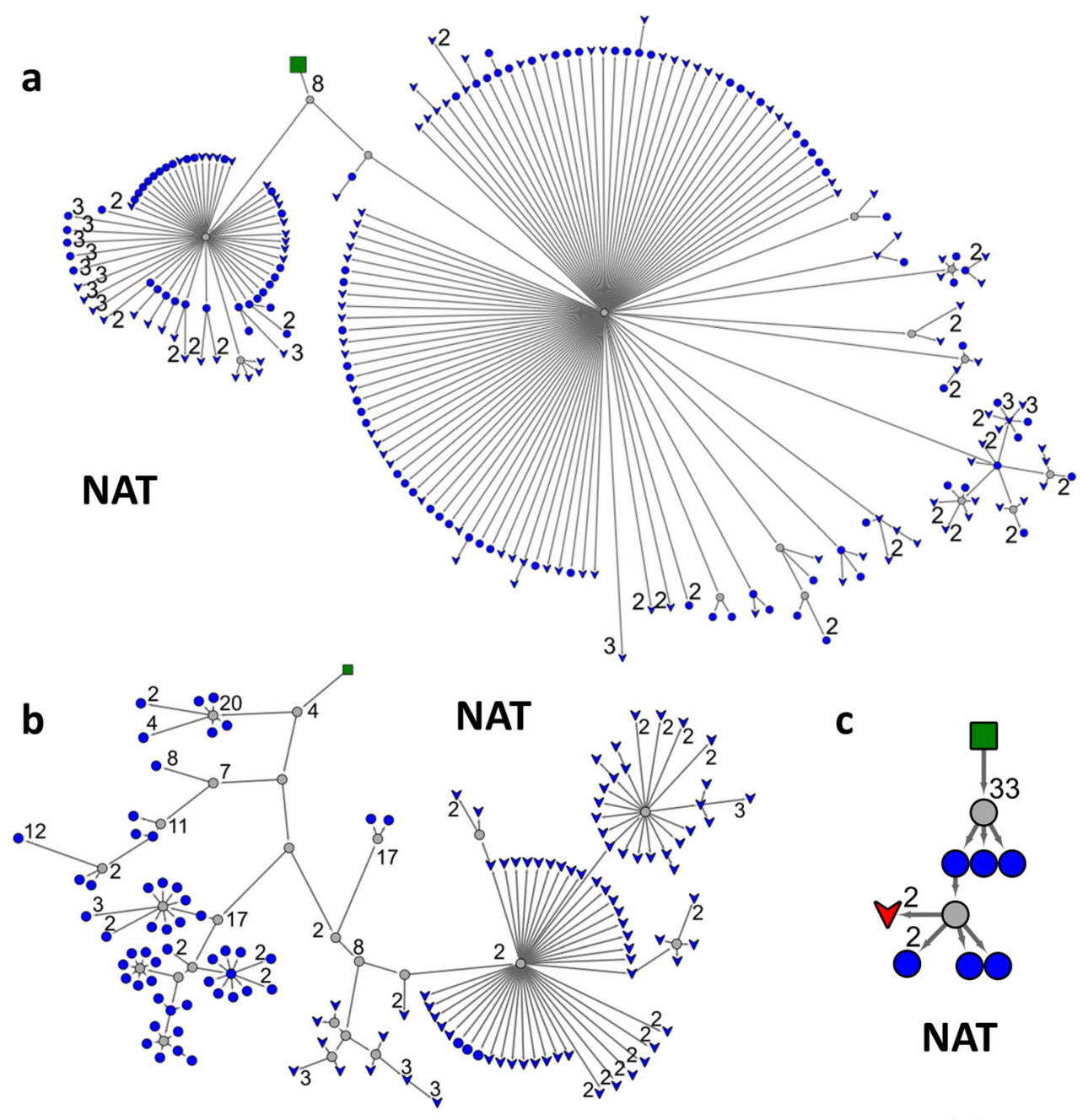

C

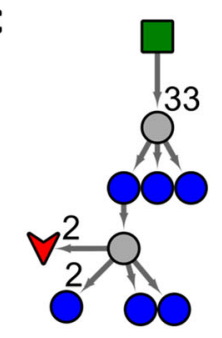

NAT

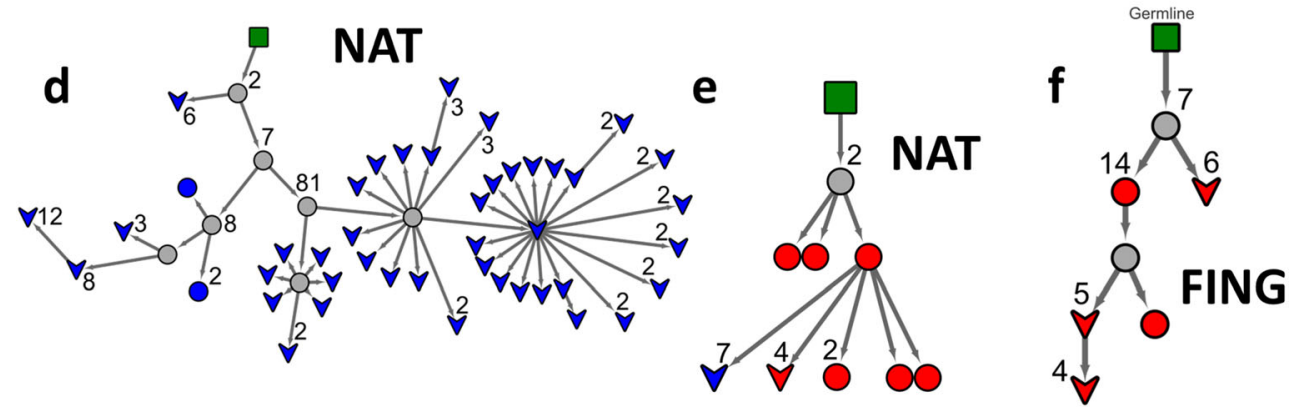

In addition, we analyzed clonal groups connecting $\mathrm{PB}$ at T0 and PB and CSF at T6 (Fig. 4g-i, Suppl. Table 4). The average number of clonal groups between both time points was significantly lower following fingolimod treatment when compared to natalizumab (fingolimod $n=7$ vs. natalizumab $n=41, p=0.03$ ). Only a very limited number of clones (fingolimod $n=$ 2 , natalizumab $n=4$ ) connected peripheral blood at T0 with CSF at T6 under both therapies. The average number of contributing sequences between both time points remained relatively stable (Fig. $4 \mathrm{~g}$ ). The distribution of clonal populations amongst PB plasmablasts and memory B cells remained stable between T0 and T6 following fingolimod treatment with only a few clones noted in the CSF DN and memory populations at T6 (Fig. 4h). In natalizumab-treated patients, mainly PB plasmablasts (20\%), PB memory B cells $(60 \%)$, and PB DN B cells (14\%) connected with PB plasmablasts (31\%), PB memory B cells (46\%), PB DN B cells $(8 \%)$, and CSF plasmablasts (11\%). The major site of clonal expansion (Fig. 4i) in fingolimod-treated patients was found in the PB at 6 months but was equally distributed between the PB and CSF in natalizumab-treated patients (Fig. 4f). 
Fig. 4 Lineage analysis of the clonal groups $(\mathrm{CG})$ identified in both peripheral blood (PB) and cerebrospinal fluid (CSF) compartments were analyzed at baseline (T0) and after 6 months (T6) of treatment. (a) The average/total number of clonal groups and the average number of contributing sequences, (b) the composition of $B$ cell subsets, and (c) the percentage of clonal populations dominated by PB or CSF sequences shared between the $\mathrm{PB}$ and $\mathrm{CSF}$ at $\mathrm{T} 0$ or $\mathrm{T} 6$ for fingolimod- and natalizumabtreated patients. (d) The average number/total number of clonal groups and the average number of contributing sequences, (e) the composition of B cell subsets, and (f) the percentage of clonal populations dominated by $\mathrm{PB}$ or CSF sequences shared between the CSF compartment at $\mathrm{T} 0$ and the CSF and PB at T6 for fingolimodand natalizumab-treated patients. (g) The average/total number of clonal groups and the average number of contributing sequences, (h) the composition of B cell subsets, and (i) the percentage of clonal populations dominated by $\mathrm{PB}$ or CSF sequences shared between the PB compartment at $\mathrm{T} 0$ and the CSF and PB at T6 for fingolimod- and natalizumabtreated patients

\section{a}

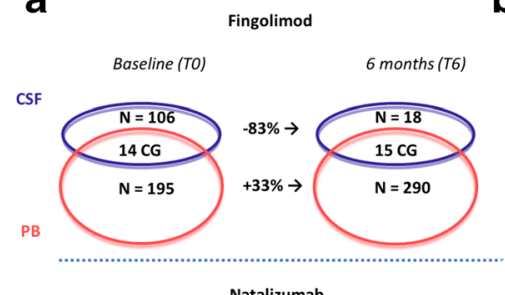

b
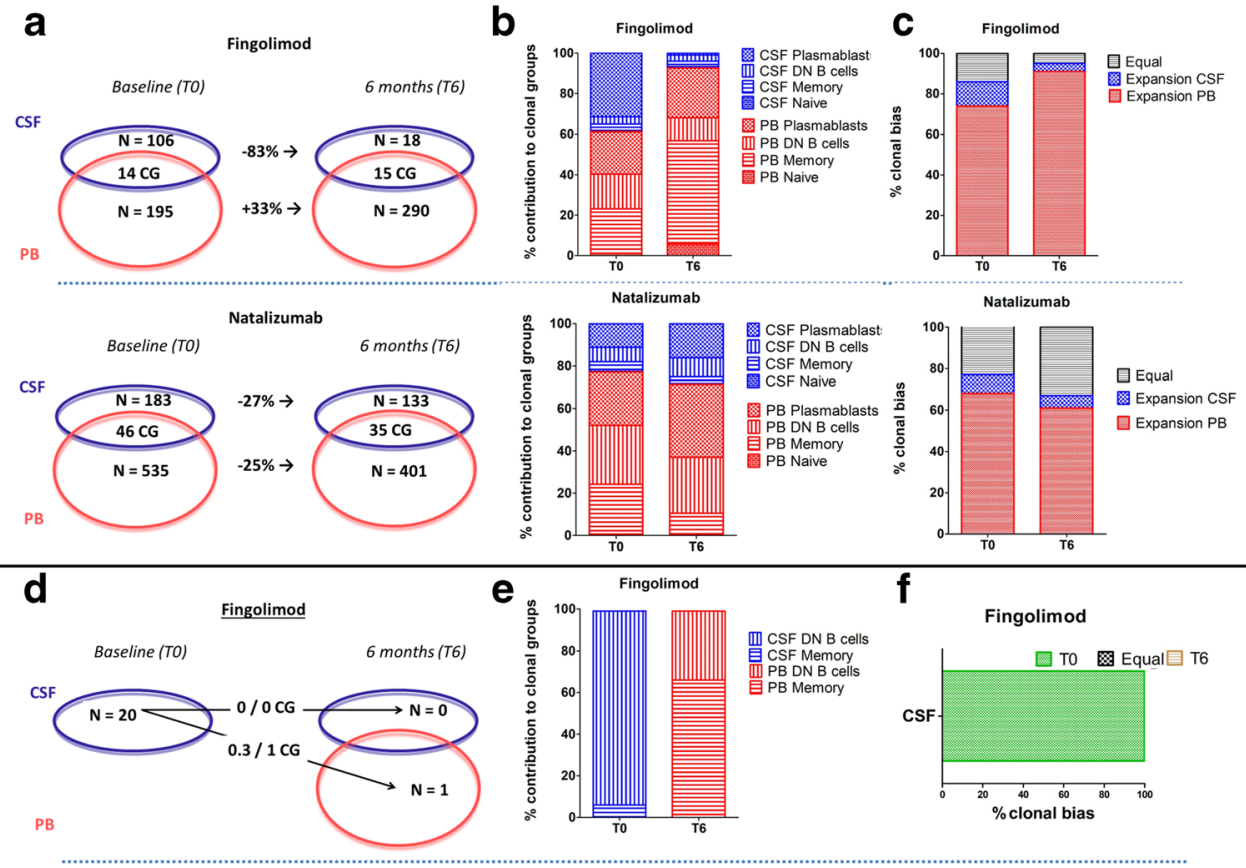

CSF DN B cells
CSF Memory II PB DN B cells
PB Memory
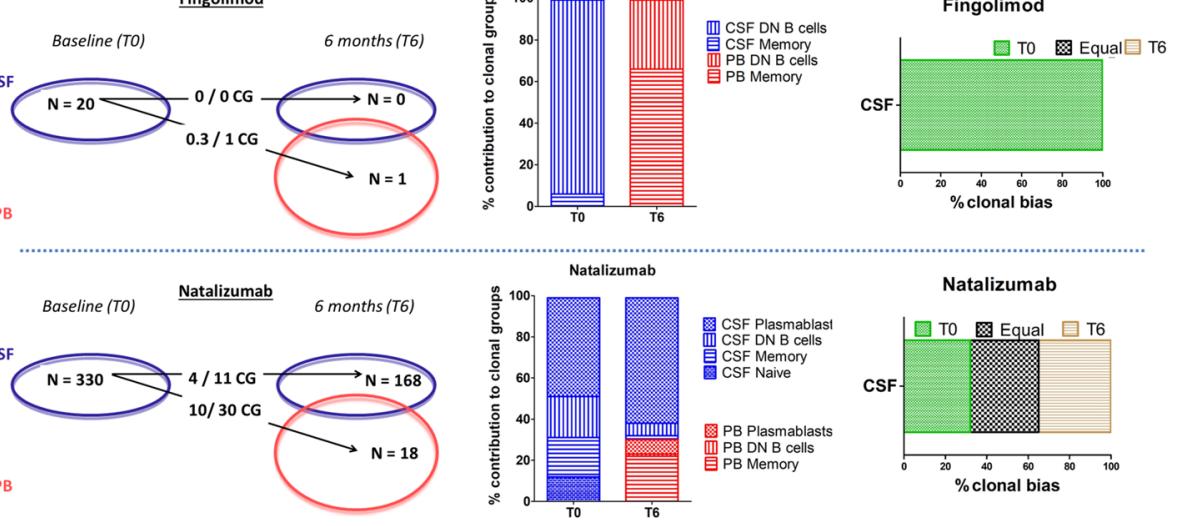

PB
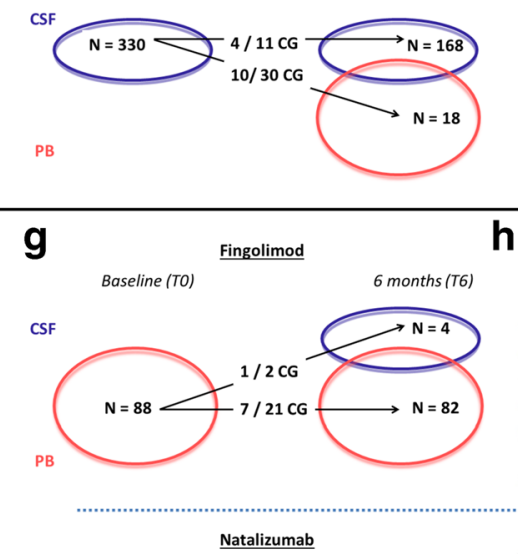

h Fingolimod
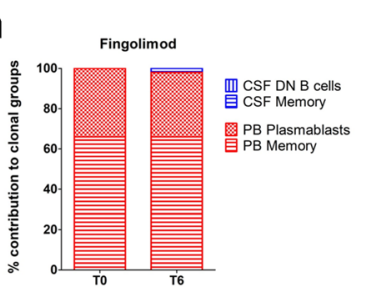

i
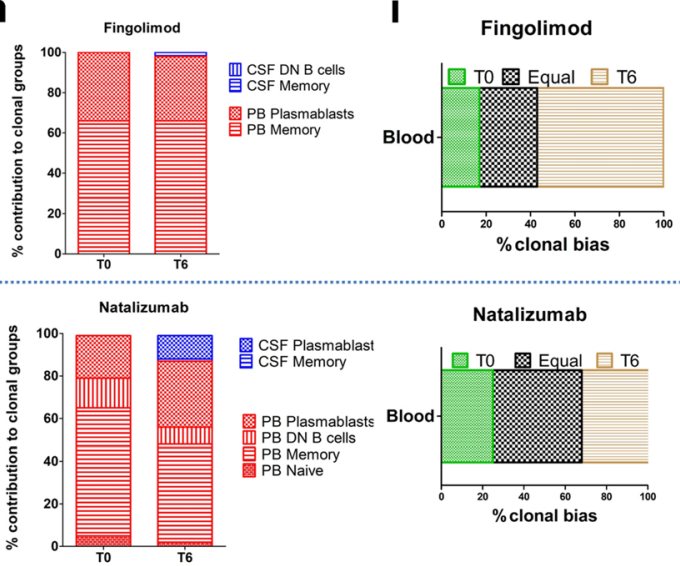

Natalizumab

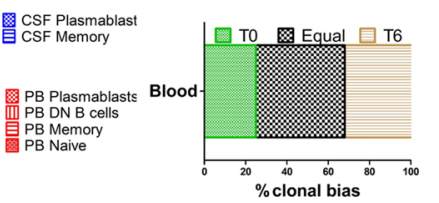

\section{Discussion}

B cells are likely to play multiple roles in the MS pathogenesis $[4,5]$. In this study, we investigated the effects of fingolimod and natalizumab on the migration and clonal expansion of $\mathrm{B}$ cells before and after 6 months of treatment in a total of $8 \mathrm{MS}$ patients by assessing Ig $\mathrm{VH}$ repertoires in the peripheral blood and CSF using next-generation sequencing. Our findings suggest that natalizumab treatment might diminish but not completely block the migration of B cells into the CSF, whereas fingolimod significantly reduces peripheral blood B cell numbers (Fig. 1) and clonal populations (Table 2) without affecting the exchange of $\mathrm{B}$ cells across the blood-brain barrier (Fig. 4). In addition, fingolimod appears to reduce B cell maturation and expansion of migratory $\mathrm{B}$ cells within the CSF compartment resulting in depletion of preexisting clonal populations.

Although our analysis was limited by patient numbers and the limited number of recoverable CSF B cells in treated patients, our study provides detailed information on the differential treatment effects of two highly effective MS therapies on $\mathrm{B}$ cell repertoires in the PB and CSF. In agreement with 
prior publications [10], CSF B cell numbers recovered from fingolimod patients remained generally stable but declined in natalizumab-treated patients. As anticipated, fingolimod reduced the number of circulating $\mathrm{B}$ cells in the $\mathrm{PB}$, whereas natalizumab resulted in an increase in PB B cells $[10,11,20$, 21]. The distinctive contraction and expansion of the circulating PB B cell population by fingolimod and natalizumab may have influenced the ability to detect related sequences in the blood and CSF compartments.

Our results at baseline (T0) are comparable to recent analyses of CSF and peripheral blood VH repertoires in untreated MS patients [22-24]. In these studies, clonal relationships were observed between CSF B cells and peripheral (classswitched) memory and double-negative B cells and plasmablasts [23], and the patterns of mutations in $\mathrm{VH}$ sequences in bicompartmental clones are consistent with migration of B cell populations across the blood-brain barrier accompanied by expansion within the CNS. In our analysis, variable degrees of overlapping sequences were identified based on the comparator population employed. Before treatment, the overlap between the PB and CSF in our 8 patients was 3.8\% using the blood repertoires as a comparator or $34 \%$ when using CSF repertoires as the comparator. In previous studies, the average percentage of overlap was lower, with only $6.3 \%$ (range $0.6-23.5 \%$ ) of the CSF B cells detected in the peripheral blood repertoire [21]. The distinctions are likely due to the different processing of Ig repertoires, the definition of clones, distinct patient populations, and different depths of mass sequencing. Similar to prior bicompartmental MS B cell repertoire analyses [22-24], lineage analyses of clonal groups between the CSF and blood compartment in our patients suggested a bidirectional exchange of B cells across the bloodbrain barrier. In contrast to previous studies [23], we also found a limited overlap of PB naïve B cells to the CSF compartment $(<10 \%)$ which could be explained by a greater depth of sequencing in our analyses; memory and DN B cells and plasmablasts from the peripheral blood all contributed approximately $30 \%$ to clonal groups emanating from PB.

After 6 months of therapy with either fingolimod or natalizumab, we observed distinct effects on the quantitative overlap of sequences and B cell lineage trees (clonal groups) between the PB and CSF compartments although results have to be interpreted with care due to the limited number of patients. Following fingolimod treatment, the percentage overlap of PB-derived sequences to the CSF repertoire increased, whereas the number of linked CSF and PB clonal groups remained stable (Fig. 4a). In contrast, natalizumab treatment reduced both the percentage overlap of PB-derived sequences to the CSF repertoire and the number of linked clonal groups (Fig. 4a). This reduction following natalizumab treatment is consistent with its known effects on restricting activated immune cell trafficking to the CNS. The high frequency of clonal groups shared between PB and CSF under fingolimod treatment suggests that despite the limited number of PB B cells, CSF migration remains unaffected. This is consistent with previous observations demonstrating a relatively high fraction of $\mathrm{B}$ cells in the CSF of patients receiving fingolimod therapy [10]. In the current study, the increased overlap between the peripheral blood and CSF B cells in fingolimodtreated patients may be magnified by the significant contraction of the circulating PB B cell population.

In addition, our results suggest that fingolimod may inhibit the lifespan of B cells or germinal center activity within the CNS. In our longitudinal analyses of CSF and blood repertoires pre- and posttreatment, we noted a lower amount of overlapping sequences and clonal populations in fingolimodtreated patients when compared to natalizumab-treated or untreated MS patients. Furthermore, detailed lineage analyses revealed that clonal expansion was significantly reduced compared to that observed following natalizumab treatment (Fig. $3)$. The limited detection of clonal groups connecting the peripheral blood compartment between $\mathrm{T} 0$ and $\mathrm{T} 6$ in fingolimod-treated patients may have been due to sequestration of PB B cells in peripheral lymphoid tissue. However, no clonal groups persisted amongst pre- and posttreatment CSF B cells under fingolimod treatment at all. Regarding these longitudinal analyses, differences in baseline characteristics may have influenced our results. Patients treated with natalizumab showed a shorter disease duration, higher cell count, and more consistent intrathecal antibody production. Nevertheless, individual baseline VH repertoires served as the comparator for longitudinal analyses after 6 months of treatment for each patient and B cell follicular structures have especially been found in patients with longer disease duration that tend to have less disease activity like in our fingolimod cohort [25]. In addition, after 6 months of fingolimod treatment, the number of clonal groups connecting the peripheral blood and CSF remained stable but showed a smaller contribution from CSF $\mathrm{B}$ cells and a larger contribution from clonally expanded peripheral blood B cells. This indicates that B cells still enter the CSF under fingolimod treatment but might not undergo further somatic hypermutation and clonal expansion within the CSF compartment (Fig. 5). Looking at the composition of B cell subsets in clonal groups between the PB and CSF compartment at $\mathrm{T} 0$ and $\mathrm{T} 6$, the percentage of CSF plasmablasts was reduced under fingolimod treatment, indicating that the occurrence of newly emerging antibody-producing plasmablasts might be inhibited by the drug. Although circulating memory B cells are decreased in the peripheral blood of fingolimod-treated MS patients, memory B cells appear to maintain an intensive exchange across the blood-brain barrier.

Our results on persisting clonal groups in the CSF of MS patients show some similarities to a recently published paper examining treated and untreated MS patients [26]. In general, the number of persisting clonal populations were lower than in our study (range 1-9) and were preferentially found in patients 
Fig. 5 Summary of treatmentassociated effects on B cell trafficking

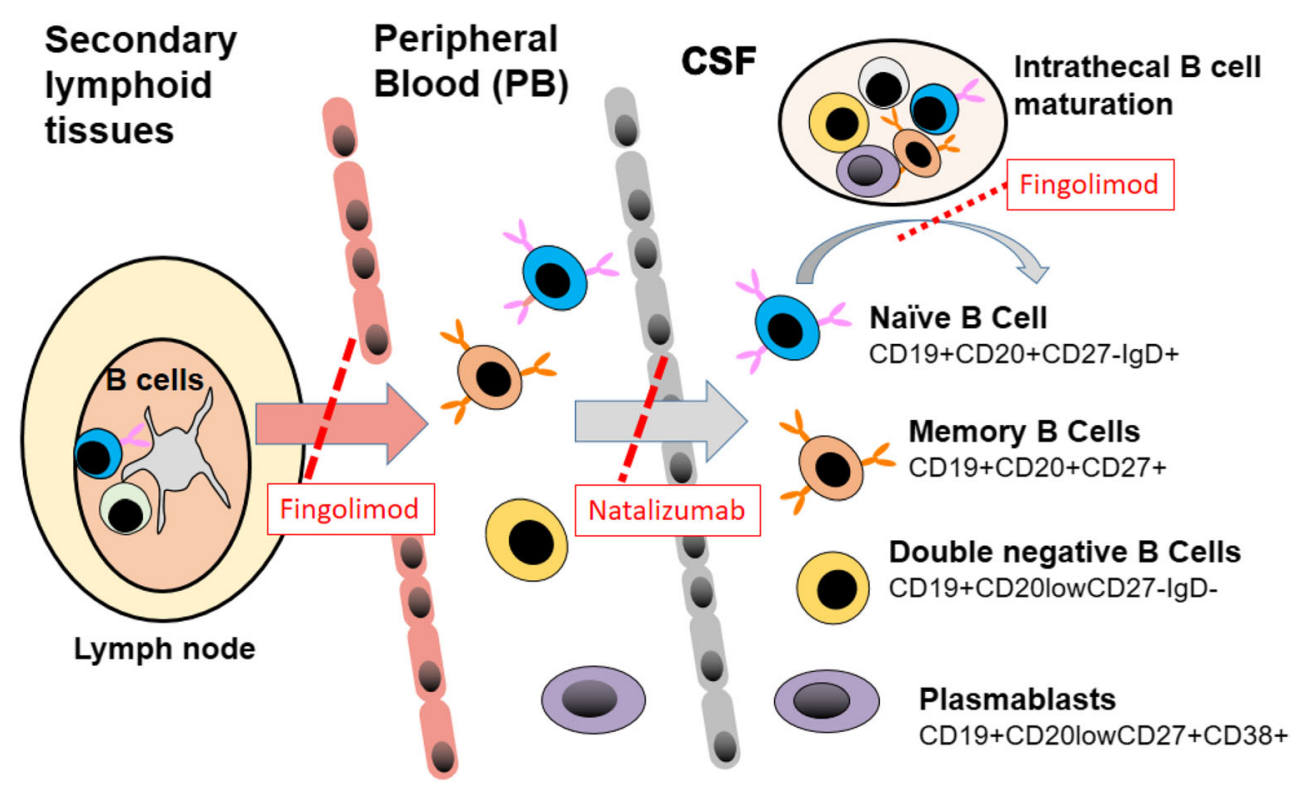

who experienced relapse during the observational period (922 months). Amongst those patients receiving natalizumab or fingolimod ( $n=2$ for each therapy), persisting clonal populations were noted in only one individual for each treatment. Both of these patients, however, showed clinical and MRI activity over an observation period of more than 12 months, making it difficult to compare with our patients who were clinically and radiologically stable over 6 months. Additional variability likely results from the limited number of patients in both studies, differences in the disease course of each patient, and limited number of CSF B cells to be sampled in natalizumab- and fingolimod-treated patients.

The combined effects of fingolimod on the PB and CSF B cell repertoires suggests that intrathecal fingolimod may reduce CNS germinal center activity. Although a role of alpha4 integrins in peripheral lymphoid tissue compartmentalization has been established in animal models [12], a similar effect of natalizumab on germinal center reactions in the CSF is unlikely. The transit of natalizumab across the blood-brain barrier as an antibody is limited and CSF concentration levels of natalizumab are 350 times less than in the serum [27]. In contrast, natalizumab is more likely to affect B cell trafficking across the blood-brain barrier in agreement with recent studies in EAE models [28]. Phosphorylated FTY720 (fingolimod) readily crosses the blood-brain barrier and is capable of interacting with CNS-resident cell populations $[29,30]$. Previous work in the mouse experimental autoimmune encephalomyelitis (EAE) model has shown that fingolimod becomes ineffective in mice selectively deficient for sphingosine-1-phosphate 1 receptors on astrocytes [31]. In addition, fingolimod ameliorates chronic progressive experimental autoimmune encephalitis mediated by astrocytes, microglia, and pro-inflammatory monocytes [30], and mice treated with fingolimod demonstrate a significant reduction of germinal center activity in peripheral lymphoid tissues [32]. To the best of our knowledge, there are no directly comparable data regarding the effects of fingolimod on CNS germinal center activity in humans.

Our analysis of the overlap between the CSF and peripheral blood B cells in MS patients under fingolimod and natalizumab treatment has several limitations. First, we evaluated only four patients in each treatment group limiting the power of our analyses and leaving some results at a descriptive level. Despite this limitation, we were still able to detect significant differences between treatment groups. Second, we observed a more intense overlap between the PB and CSF compartment at baseline in MS patients receiving natalizumab. Although these differences were not significant, physician biases and disease activity may have biased patient selection into the treatment groups, thereby influencing the results. Indeed, natalizumab-treated patients showed a shortened disease duration and lower age; however, EDSS values were only slightly higher in the natalizumab cohort (Table 1). In addition, oligoclonal bands were detectable in all natalizumab-treated patients, whereas two fingolimod-treated patients did not show evidence for an intrathecal Ig synthesis at baseline and after 6 months of treatment which might have also influenced our results. Nevertheless, we were able to generate representative repertoires in both treatment groups, and our subsequent analyses, normalized by the total number of obtained sequences, were consistent when comparing the total number of overlapping sequences with the detailed analyses of clonal groups. Third, although lineage analysis provides a unique tool to study $B$ cell trafficking in $\mathrm{MS}$, mass sequencing of B cell Ig repertoires only allows a snapshot of the $\mathrm{B}$ cell repertoire at a certain time and does not fully represent the dynamic relationship amongst transiting B cells. Results may be skewed by the process of repertoire 
reconstruction. Although we optimized our protocol to obtain a maximum recovery of Ig sequences from B cells, efficient amplification of $\mathrm{VH}$ sequences from memory and naïve $\mathrm{B}$ cells was challenging, and despite safeguards such as highfidelity PCR and UMIs, sequencing errors and overamplification of transcripts may result in artificial, or overrepresented, B cell clusters/clones. For instance, we observed roughly $50 \%$ of naïve B cells in clones containing a small number of mutations from germline (3.5 sequences/ clone). These small clones could be the product of sequencing error or could represent activated naïve B cells [33]. Our approach did not allow us to discriminate different $\mathrm{B}$ cells with nucleotide-identical RNA/cDNA strands, because the UMI was introduced after the cDNA synthesis step. We have worked to minimize any such bias by validating each individual Ig lineage tree according to B cell subsets, distance to germline, and time point of analysis.

In summary, our results confirm that natalizumab inhibits but does not completely block the migration of $\mathrm{B}$ cells across the blood-brain barrier. Natalizumab, however, does not impair B cell germinal center activity within the CNS. Although the exchange of PB B cells across the blood-brain barrier does not seem to be inhibited following fingolimod treatment, B cell numbers are significantly reduced in the peripheral blood. More importantly, B cells seem to be inhibited in their ability to undergo further maturation within the CNS. Especially in the context of progressive MS, in which ongoing germinal center activity within the CNS may be compartmentalized [25], CNS penetration of sphingosine-1-phosphate (S1P) inhibitors may offer a unique avenue to modulate the intrathecal B cell response. Although fingolimod treatment did not reach primary endpoints in the INFORMS trial in primary progressive MS, MRI parameters of disease activity showed a significant reduction in the number of T2 lesions [34]. Recently, siponimod, a more selective S1P receptor functional antagonist demonstrated benefit in the treatment of SPMS [35] and was recently approved by the FDA. Thus, S1P receptor inhibitors may have significant effects on trapped CNS inflammation in progressive MS patients. Further studies of different S1P receptors and their effects on B cell maturation within the $\mathrm{CSF} / \mathrm{CNS}$ in a larger patient collective will be necessary to fully understand the effects of this class of compounds on the B cell compartment.

Supplementary Information The online version contains supplementary material available at https://doi.org/10.1007/s13311-020-00975-7.

Acknowledgments This work was funded by the National Institutes of Health (R01EY022936 to JLB), the National MS Society, and an independent investigator grant from Novartis Pharmaceutical (JLB). Bernhard Hemmer received support from the Deutsche Forschungsgemeinschaft (DFG, German Research Foundation) under Germany's Excellence Strategy within the framework of the Munich Cluster for Systems Neurology (EXC 2145 SyNergy - ID 390857198) and the EU project MultipleMS. M. C. Kowarik was supported by the Deutsche Forschungsgesellschaft (DFG, Ko 4367/1-1) and SyNergy.
Required Author Forms Disclosure forms provided by the authors are available with the online version of this article.

\section{References}

1. Krumbholz M, Meinl E. B cells in MS and NMO: pathogenesis and therapy. Semin Immunopathol. 2014;36:339-350.

2. Gasperi C, Salmen A, Antony G, et al. Association of intrathecal immunoglobulin $\mathrm{G}$ synthesis with disability worsening in multiple sclerosis. JAMA Neurol. 2019;76(7):841-849.

3. Kappos L, Li D, Calabresi PA, et al. Ocrelizumab in relapsingremitting multiple sclerosis: a phase 2 , randomised, placebo-controlled, multicentre trial. Lancet. 2011;378:1779-1787.

4. Montalban X, Hauser SL, Kappos L, et al. Ocrelizumab versus placebo in primary progressive multiple sclerosis. N Engl J Med. 2017;376(3):209-220.

5. Hauser SL, Bar-Or A, Comi G, et al. Ocrelizumab versus interferon beta-1a in relapsing multiple sclerosis. N Engl J Med. 2017;376(3): 221-234.

6. Kappos L, Radue EW, O'Connor P, et al. A placebo-controlled trial of oral fingolimod in relapsing multiple sclerosis. N Engl J Med. 2010;362:387-401.

7. Polman $\mathrm{CH}, \mathrm{O}^{\prime}$ Connor PW, Havrdova E, et al. A randomized, placebo-controlled trial of natalizumab for relapsing multiple sclerosis. N Engl J Med. 2006;354:899-910.

8. Mandala S, Hajdu R, Bergstrom J, et al. Alteration of lymphocyte trafficking by sphingosine-1-phosphate receptor agonists. Science. 2002;296:346-349.

9. Matloubian M, Lo CG, Cinamon G, et al. Lymphocyte egress from thymus and peripheral lymphoid organs is dependent on S1P receptor 1. Nature. 2004;427:355-360.

10. Kowarik MC, Pellkofer HL, Cepok S, et al. Differential effects of fingolimod (FTY720) on immune cells in the CSF and blood of patients with MS. Neurology. 2011;76:1214-1221.

11. Stuve O, Marra CM, Jerome KR, et al. Immune surveillance in multiple sclerosis patients treated with natalizumab. Ann Neurol. 2006;59:743-747.

12. Lu TT, Cyster JG. Integrin-mediated long-term $B$ cell retention in the splenic marginal zone. Science. 2002;297(5580):409-12.

13. Kowarik MC, Astling D, Gasperi C, et al. CNS aquaporin-4specific B cells connect with multiple B-cell compartments in neuromyelitis optica spectrum disorder. Ann Clin Transl Neurol. 2017;4:369-380

14. Kivioja T, Vähärautio A, Karlsson K, et al. Counting absolute numbers of molecules using unique molecular identifiers. Nature methods. 2012;9:72-74.

15. Van der Heiden JA, Yaari G, Uduman M, et al. pRESTO: a toolkit for processing high-throughput sequencing raw reads of lymphocyte receptor repertoires. Bioinformatics. 2014;30:1930-1932

16. Alamyar E, Duroux P, Lefranc MP, Giudicelli V. IMGT((R)) tools for the nucleotide analysis of immunoglobulin (IG) and T cell receptor (TR) V-(D)-J repertoires, polymorphisms, and IG mutations: IMGT/V-QUEST and IMGT/HighV-QUEST for NGS. Methods Mol Biol. 2012;882:569.

17. Barak M, Zuckerman NS, Edelman H, Unger R, Mehr R. IgTree: creating immunoglobulin variable region gene lineage trees. J Immunol Methods. 2008;338(1-2):67-74

18. Brezinschek HP, Foster SJ, Brezinschek RI, Dorner T, DomiatiSaad R, and Lipsky PE. Analysis of the human VH gene repertoire. J. Clin. Invest. 1998.99: 2488-2501.

19. Owens GP, Winges KM, Ritchie AM, et al. VH4 gene segments dominate the intrathecal humoral immune response in multiple sclerosis. J Immunol. 2007;179(9):6343-51. 
20. Traub JW, Pellkofer H, Grondey K, et al. Natalizumab promotes activation and pro-inflammatory differentiation of peripheral $\mathrm{B}$ cells in multiple sclerosis patients. J Neuroinflammation. 2019;16(1):228.

21. Kemmerer CL, Pernpeintner V, Ruschil C et al. Differential effects of disease modifying drugs on peripheral blood B cell subsets: a cross sectional study in multiple sclerosis patients treated with interferon- $\beta$, glatiramer acetate, dimethyl fumarate, fingolimod or natalizumab. PlosOne. In press.

22. von Büdingen HC, Kuo TC, Sirota M, et al. B cell exchange across the blood-brain barrier in multiple sclerosis. J Clin Invest. 2012;122(12):4533-43.

23. Palanichamy A, Apeltsin L, Kuo TC, et al. Immunoglobulin classswitched B cells form an active immune axis between CNS and periphery in multiple sclerosis. Sci Transl Med. 2014;6(248): 248 ra106.

24. Stern JN, Yaari G, Vander Heiden JA, et al. B cells populating the multiple sclerosis brain mature in the draining cervical lymph nodes. Sci Transl Med. 2014;6(248):248ra107.

25. Serafini B, Rosicarelli B, Magliozzi R, Stigliano E, Aloisi F. Detection of ectopic B-cell follicles with germinal centers in the meninges of patients with secondary progressive multiple sclerosis. Brain Pathol. 2004;14(2):164-74.

26. Greenfield AL, Dandekar R, Ramesh A, et al. Longitudinally persistent cerebrospinal fluid $\mathrm{B}$ cells can resist treatment in multiple sclerosis. JCI Insight. 2019;4(6).

27. Harrer A, Pilz G, Wipfler P, et al. High interindividual variability in the CD4/CD8 $\mathrm{T}$ cell ratio and natalizumab concentration levels in the cerebrospinal fluid of patients with multiple sclerosis. Clin Exp Immunol. 2015;180(3):383-92.

28. Lehmann-Horn K, Sagan SA, Bernard CC, Sobel RA, Zamvil SS. B-cell very late antigen-4 deficiency reduces leukocyte recruitment and susceptibility to central nervous system autoimmunity. Ann Neurol. 2015;77(5):902-8.

29. Lee CW, Choi JW, Chun J. Neurological S1P signaling as an emerging mechanism of action of oral FTY720 (fingolimod) in multiple sclerosis. Arch Pharm Res. 2010;33(10):1567-74.

30. Rothhammer V, Kenison JE, Tjon E, et al. Sphingosine 1phosphate receptor modulation suppresses pathogenic astrocyte activation and chronic progressive CNS inflammation. Proc Natl Acad Sci U S A. 2017 Feb 6.

31. Choi JW, Gardell SE, Herr DR, et al. FTY720 (fingolimod) efficacy in an animal model of multiple sclerosis requires astrocyte sphingosine 1-phosphate receptor 1 (S1P1) modulation. Proc Natl Acad Sci U S A. 2011;108:751-756.

32. Han S, Zhang X, Wang G, et al. FTY720 suppresses humoral immunity by inhibiting germinal center reaction. Blood. 2004;104(13):4129-33.

33. Tipton CM, Fucile CF, Darce J, et al. Diversity, cellular origin and autoreactivity of antibody-secreting cell population expansions in acute systemic lupus erythematosus. Nat Immunol. $2015 \mathrm{Jul} ; 16(7)$ : 755-65.

34. Lublin F, Miller DH, Freedman MS, et al. Oral fingolimod in primary progressive multiple sclerosis (INFORMS): a phase 3, randomised, double-blind, placebo-controlled trial. Lancet. 2016;387(10023):1075-84.

35. Kappos L, Bar-Or A, Cree BAC, et al. Siponimod versus placebo in secondary progressive multiple sclerosis (EXPAND): a doubleblind, randomised, phase 3 study. Lancet. 2018;391(10127):12631273.

Publisher's Note Springer Nature remains neutral with regard to jurisdictional claims in published maps and institutional affiliations. 